\title{
An Investigation into the Effect of Patient Safety Indicators on Patient Satisfaction: A Case Study of Shooshtar Maternity Hospital in Shiraz
}

\author{
Ayeh Negahbani', Shaghayegh Vahdat ${ }^{2 *}$ and Somayeh Hessam ${ }^{3}$ \\ 'Master of Science, Department of Healthcare Management, Marvdasht Branch, Islamic Azad University, Marvdasht, Iran \\ ${ }^{2}$ Assistant Professor, Department of Health Services Administration, South Tehran Branch, Islamic Azad University, \\ Tehran, Iran; sha_vahdat@yahoo.com \\ ${ }^{3}$ Assistant Professor, Department of Health Services Administration, South Tehran Branch, Islamic Azad University, Tehran, Iran
}

\begin{abstract}
Today, one of the main healthcare issues is maintaining the safety of patients in healthcare centers. Patient safety is a global health concern that affects patients in all countries, whether developed or developing countries. For this purpose, the present study investigates the effect of patient safety indicators on patient satisfaction at Shooshtar Maternity Hospital in Shiraz. Data obtained from monthly reports of the hospital during 2015-17 were used to study the research hypotheses. The hypotheses were analyzed using regression test and SPSS. Results showed that infection control parameters, anesthetic complications, blood transfusion complications, removal of surgical wound, post-surgery bleeding, accidental perforation, surgical wound infections, normal delivery with or without instrumentation and birthrate had a significant effect on patient satisfaction.
\end{abstract}

Keywords: Patient Safety Indicators, Patient Satisfaction, Shooshtar Maternity Hospital of Shiraz

\section{Introduction}

Today, one of the main healthcare issues is maintaining the safety of patients in healthcare centers. Patient safety is a global health concern that affects patients in all countries, whether developed or developing countries. In fact, one of the priorities of the health system is to improve the quality of treatment, patient safety and other healthcare programs ${ }^{1}$. When we talk about patient safety, we're really talking about how hospitals and other health care organizations protect their patients from errors, injuries, accidents, and infections. While many hospitals are good at keeping their patients safe, some hospitals aren't. The International Agency for Patient Safety (2003) defined patient safety as a process by which the hospital provides more safe the patients. Therefore, patient safety includes:

- Risk Assessment

- Identification and management of patient-related risks

- Reporting and analyzing the incidents

The ability to learn and track incidents and implement solutions to minimize the error ${ }^{2}$.
Patient safety indicators are measures that directly or indirectly prevent adverse incidents and monitor the quality and outcomes. By monitoring these indicators, it would be possible to design a program to improve the safety and quality of services ${ }^{3}$. Patient safety practices have been defined as "those that reduce the risk of adverse events related to exposure to medical care across a range of diagnoses or conditions". This definition is concrete but quite incomplete, because so many practices have not been well studied with respect to their effectiveness in preventing or ameliorating harm. Healthcare is inevitably associated with an increased risk of patient safety and health threats. Patients need to be ensured that healthcare services will not endanger their health. These services should provide the best conditions and indicators, and the latest scientific and clinical evidence. Therefore, patient safety programs affect both physical and mental health of the patients and may either increase or decrease their satisfaction. Given the growing importance of customers in determining the quality of products and services offered in the market, many organizations have been paying increasing attention to customer satisfaction criteria. Organizations aim to provide better services for the patients. Organizations and hospitals

*Author for correspondence 
follow the same objectives and have taken some important measures in this regard. Accordingly, patient satisfaction is one of the important outcomes of healthcare quality. It is regarded as one of the most important indicators for the effectiveness of services provided in healthcare centers ${ }^{4}$. Given the patients' specific circumstances, it is inappropriate to call them customers when referring to a health facility or hospital. Patient satisfaction is to receive appropriate healthcare services in order to fulfill one's inner needs and desires. In addition, satisfaction has a significant role in maintaining health and preventing diseases. Patient satisfaction has been a very important issue for researchers involved in health-care systems for the last few decades. This is because the patient is the most important person in the entire hospital setup. Thus, increasing satisfaction will improve the physical and mental health of the patient ${ }^{6}$. Accordingly, patient satisfaction is an important issue which improves the quality of health care in any country ${ }^{7}$. In the present study, we have examined one of the important factors affecting patient satisfaction in order to determine to what extent patient safety can provide patient satisfaction.

\section{Materials and Methods}

This applied research used descriptive methods to collect the data. Given the relationship between the variables studied, this can be regarded as a correlational research. The data collection process was also based on library methods (papers, books and theses). In order to collect the data related to the research variables, information about satisfaction indicators and safety indicators of the patient in Shooshtar Maternity
Hospital in Shiraz (2015-2017) was used. Descriptive and inferential methods were used to analyze the data. Descriptive methods provide a summary of measurements about the sample, while inferential methods allow us to judge the total population based on the results of the sample and come to a general conclusion. To investigate the relationship between the variables, regression test and SPSS were used.

\section{Findings}

According to the Table, bedsore index, falling from the bed, post-surgery complications index, mortality rate in postnatal hospital complications, venous thrombosis or post-surgery pulmonary embolism and MI mortality rate have no significant effect on patient satisfaction.

Infection control index, blood transfusion index, wound closure index, post-surgery complications index, mortality rate in hospital complications, MI mortality rate and birth index have a significant effect on patient satisfaction.

\section{Discussion}

Regarding the importance of patient safety and patient safety indicators in healthcare centers and hospitals, studies have shown that therapeutic measures are usually associated with dangerous situations. According to the World Health Organization (WHO), tens of millions of people die or become disabled annually due to clinical errors and unsafe healthcare processes. Clinical errors are one of the most important health

\begin{tabular}{|l|l|l|l|l|l|}
\hline Independent Variable & Dependent Variable & $\begin{array}{l}\text { Correlation } \\
\text { Coefficient }\end{array}$ & $\begin{array}{l}\text { T } \\
\text { Level }\end{array}$ & $\begin{array}{l}\text { Determination } \\
\text { Coefficient }\end{array}$ \\
\hline Falling from the bed & Patient Satisfaction & -0.218 & -1.304 & 0.201 & 0.048 \\
\hline Bedsore & Patient Satisfaction & -0.050 & -0.290 & 0.773 & 0.002 \\
\hline Blood transfusion & Patient Satisfaction & -0.019 & -5.022 & 0.000 & 0.120 \\
\hline Infection control & Patient Satisfaction & 0.232 & 5.146 & 0.000 & 0.054 \\
\hline Anesthesia complications & Patient Satisfaction & -0.252 & -4.494 & 0.000 & 0.630 \\
\hline Blood transfusion complications & Patient Satisfaction & -0.109 & -5.22 & 0.000 & 0.120 \\
\hline Wound complications during the surgery & Patient Satisfaction & -0.554 & -11.029 & 0.000 & 0.308 \\
\hline mortality rate in post-delivery complications & Patient Satisfaction & -0.292 & -1.173 & 0.084 & 50.08 \\
\hline Accidental perforation & Patient Satisfaction & -0.366 & -6.490 & 0.000 & 0.134 \\
\hline Post-surgery complications & Patient Satisfaction & -0.049 & -0.278 & 0.776 & 0.002 \\
\hline venous thrombosis or post-surgery pulmonary embolism & Patient Satisfaction & -0.047 & -0.027 & 0.784 & 0.002 \\
\hline Post-surgery wound complications & Patient Satisfaction & -0.611 & -11.459 & 0.000 \\
\hline MI mortality rate & Patient Satisfaction & -0.019 & -0.112 & 0.911 & 0.437 \\
\hline Natural delivery & Patient Satisfaction & -0.761 & -15.265 & 0.000 \\
\hline Birthrate & Patient Satisfaction & -0.353 & -2.191 & 0.035 & 0.000 \\
\hline
\end{tabular}


challenges in all countries. Studies have shown that on average $10 \%$ of all hospital admissions are injured patients in varying degrees and it is estimated that up to $75 \%$ of the errors can be prevented. Accordingly, patient safety failures result in serious medical hazards, affecting the patient satisfaction and his fellows. On the other hand, patient satisfaction has become an important issue in recent years in all countries. Patient satisfaction emphasizes on the necessity of compliance and follow up of the treatment process, the vulnerability of patients and the need for comprehensive support. The increased public awareness has, in recent years, increased the patients' expectations of receiving services. Toinstin (2010) believes that in today's world, the importance of measuring patients' satisfaction is one of the basic criteria for determining the quality of healthcare services. Therefore, it is important to examine the factors affecting patient satisfaction. An investigation into the research literature showed not all aspects and factors affecting patient satisfaction have been studied. Accordingly, this study aimed to investigate the effect of patient safety on patient satisfaction.

The results indicate that the patient safety culture measures and their dimensions have been evaluated by the Liker's Scale. The results were similar to those of Mohebifar et al. ${ }^{9}$, Abdi et al. ${ }^{10}$, Mahfoozpour et al. ${ }^{11}$, Izadi et al. ${ }^{12}$, Moghri et al. ${ }^{13}$, and Yaqoobifar et al. According to these researchers, patient safety was in an acceptable status. According to Mahfoozpour et al., patient safety was evaluated in the educational hospitals affiliated to Shahid Beheshti University of Medical Sciences. Moghri et al. reported that patient safety was lower than almost all other criteria in general hospitals affiliated to Tehran University of Medical Sciences. Yaghoubifar et al. reported that patient safety in Sabzevar hospitals was moderate. According to Abdi et al., patient safety in the elite hospitals of Tehran University of Medical Sciences was low to moderate. Finally, Mohebirf et al. argued that the average patient safety was higher than the average according to the staff of hospitals in Tehran. Mohebifar et al. believed that this can be attributed to the implementation of clinical governance and credibility indicators in Iran that have promoted the patient safety.

In order to improve the patient safety based on the data analysis, the following recommendations can be considered: (A) It is necessary to take serious actions in order to encourage the nurses to perform their tasks efficiently, using in-service training. Nurses need to treat their colleagues with respect and help others. (B) In order to strengthen employee affairs, hospitals should have enough nurses who can perform efficiently under great pressure. To this end, managers should encourage the nurses to keep working. (C) To strengthen the processes, standardized procedures for patient care should be used. (D) In order to strengthen the education and skills, nurses should learn what they need in the hospital to care for patients through training so that they can improve their skills. More importantly, they have to get involved in the working processes in order to make appropriate decisions. (E) Nurses should not be afraid to report their mistakes and do not feel at risk when reporting. (F) The supervisors should examine all the factors to determine the root cause of the mistakes. One needs to avoid blaming and punishing nurses. (G) The supervisors should be mindful of patient safety issues, respect nurses' views on patient safety and use $\mathrm{d}$ encouraging methods as they do their job properly. (H) In order to enhance the overall patient safety perceptions, hospitals should provide an appropriate space and interior design and a safe environment for patients. (I) Managers should ask nurses to increase the patient safety culture in the hospital. In this regard, managers should consider nurses' suggestions for improving patient safety. They should also monitor the patient safety by continuously attending different departments of the hospital. (J) In order to enhance organizational learning, hospitals should continue to innovate in the workplace to improve patient safety and try to make changes easily to. In addition, the same mistakes should be avoided. (K) In order to enhance the patient delivery, let nurses know what they need before they first take care of patients. In addition, information should be provided to nurses when patients are transferred to hospitals. They will also be asked to make the right decision when a change is made in the patient safety program. (L) In order to improve the feedback, nurses should be advised to report timely when patients are injured. On the other hand, it is also necessary to talk about preventing what has happened over and over and how it protects patients from harm. (M) In order to improve the freedom of communication, nurses' feedback and suggestions should be evaluated. In this regard, nurses' opinions should be considered. In addition, they should talk freely about problems within the hospital. Patients should be encouraged to work in order to increase their job satisfaction. They have to feel that they are valuable not only for the units, but also the hospital they are working in. Not only do nurses themselves have to prioritize patients' safety, but also try to make their clinical teams and their work hospitals more aware of the patient safety. On the other hand, nurses should also have a positive view of the management's concern about budget constraints. In addition, they should be serious about expressing concerns about patient safety issues. Finally, nurses should believe that managers are sensitive to the reports.

The results show that there is a significant relationship between patients' job satisfaction and the patient safety indicators. Moreover, there is a direct relationship between patients' job satisfaction and patient safety. In other words, the greater the patients' job satisfaction, the better the patient safety culture. As a result, the patient safety culture increases to a great extent. 


\section{References}

1. Salehi M. Explaining factors affecting nurses' job satisfaction and patient safety culture in Neyshabour hospitals, Master's thesis, Islamic Azad University, Neyshabur Branch. 2015.

2. Graban M. Lean hospitals: improving quality, patient safety, and employee satisfaction. CRC Press. 2015. https://doi.org/10.1201/ b11740

3. Rosen AK, Itani KM, Cevasco M, Kaafarani HM, Hanchate A, Shin M \& Borzecki A. Validating the patient safety indicators in the Veterans Health Administration: do they accurately identify true safety incidents. Medical care. 2012; 50(1):74-85. https://doi. org/10.1097/MLR.0b013e3182293edf PMid:21993057

4. Julian S, Giyoori A, Taavoni S, Bohrani N, Rezapour R. Patient Satisfaction Rate of Nursing Services in Educational Hospitals in Iran. Nursing Research. 2007; 2(6-7):44-57.

5. Journal of the Medical Council of the Islamic Republic of Iran. 21(4):266-70.

6. Khodam H, Jooybari L, Sangago A. The Effect of Continuing Education on the Quality of Nursing Reports. Journal of Gorgan University of Medical Sciences. 2006; 3(8):65.

7. Saraei M, Azadehdel M, Jabbari Sadodi F. An Investigation into the Relationship between Nurses' Job Satisfaction and Patient Safety in the Hospitals of Rasht. Journal of Health and Safety. 2016; 6(3):103-91.

8. Tustin N. The role of patient satisfaction in online health information seeking. Journal of Health Communication.
2010; 15(1):3-17. https://doi.org/10.1080/10810730903465491 PMid:20390974

9. Mohebi Far R, Alijanzade M, Safari Variani A, Khoshtarkib H, Ghanati E, Teymouri F, Zakaria Kiaei M, Ziaeiha M. Studying patient safety culture from the viewpoint of staffs in educational hospitals in Tehran City. Journal of Health and Safety at Work. 2015; 5(1):57-65.

10. Abdi Z, Maleki MR. Khosravi A. Perceptions of patient safety culture among staff of selected hospitals affiliated to Tehran University of Medical Sciences. Journal of the Iranian Institute for Health Sciences Research. 2011; 10(4):411-9. [in Persian].

11. Mahfoozpour S, Ainy E, Mobasheri F, Faramarzi A. Patients' safety culture status among educational hospitals of Shahid Beheshti University of Medical Sciences in 2011. Pajoohandeh Journal. 2012; 17(3):134-41. [in Persian].

12. Izadi AR, Drikvand J, Ebrazeh A. The Patient Safety Culture in Fatemeh Zahra Hospital of Najafabad, Iran. Journal of Health Information Management. 2013; 9(6):895-907. [In Persian].

13. Moghri j, Akbari Sari A, Rahimi Forooshani A, Arab M. Patient safety culture status in general hospitals affiliated to Tehran University of Medical Sciences. Hakim Research Journal. 2013; 16(3):243-50. [in Persian].

14. Yaghobi Far MA, Takbiri A, Haghgoshaye E. Tabarraye. The survey of patient safety culture and recognizing its weaknesses and strengths in Sabzevar hospitals: 2011. Journal of Sabzevar University of Medical Sciences. 2012; 20(2):154-64. [Text in Persian]. 